\section{Case Report}

Korean J Transplant 2020;34:109-113

https://doi.org/10.4285/kjt.2020.34.2.109

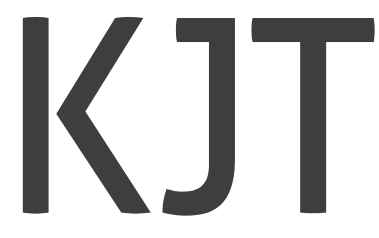

pISSN 2671-8790

elSSN 2671-8804

\title{
Successful ABO-incompatible living donor liver transplantation using splenectomy and intravenous immunoglobulin in high isoagglutinin titer patients
}

\author{
Boram Lee, Jai Young Cho, Hae Won Lee, YoungRok Choi, Yoo-Seok Yoon, \\ Ho-Seong Han
}

Department of Surgery, Seoul National University Bundang Hospital, Seoul National University College of Medicine, Seongnam, Korea

Received March 3, 2020

Revised April 21, 2020

Accepted April 22, 2020

Corresponding author: Jai Young Cho Department of Surgery, Seoul National University Bundang Hospital, 82 Gumiro 173beon-gil, Bundang-gu, Seongnam 13620 , Korea

Tel: +82-31-787-7098

Fax: +82-31-787-4078

E-mail: jychogs@gmail.com

(c) The Korean Society for Transplantation This is an Open Access article distributed under the terms of the Creative Commons Attribution Non-Commercial License (http://creativecommons.org/licenses/ by-nc/4.0/) which permits unrestricted non-commercial use, distribution, and reproduction in any medium, provided the original work is properly cited.
The role of the isoagglutinin (IA) titer in liver transplantation (LT) is still not well defined, but the general belief is that a higher titer may result in a higher risk of rejection in ABO-incompatible living donor LT. To reduce the IA titer by 1:16 or lower, plasmapheresis is usually performed before transplantation. However, there is no established protocol for patients for whom plasmapheresis has failed before reaching the target IA titers. Here, we report the cases of three patients who show high baseline IA titers and have failed plasmapheresis: no-response to plasmapheresis, allergic reaction associated with plasmapheresis, and anaphylactic reaction to platelet transfusion. For various reasons, after several plasmapheresis procedures, IA titers were not effectively reduced. In these patients, splenectomy and intravenous immunoglobulin $(0.8 \mathrm{~g} / \mathrm{kg}$, from the anhepatic phase to 2 days after transplantation) were carried. The protocol biopsy on postoperative day 7 showed no histologic evidence of meaningful acute rejection. The main aim of this work is to demonstrate that we can apply this protocol to patients who have high baseline IA titers and have failed plasmapheresis. Furthermore, this report is enhanced to promote to the transplant community this approach with this type of recipient.

Keywords: Liver transplantation; Plasmapheresis; Graft rejection; Splenectomy; Immunoglobulins, intravenous

\section{INTRODUCTION}

Since the introduction of desensitization (DSZ) with rituximab (RTX; anti-CD20 monoclonal antibody; Rituxan \& MabThera, Roche, Switzerland), graft survival after ABO-incompatible (ABOi) living donor liver transplantation (LDLT) has been gradually improving. However, there is no standardized DSZ protocol for preventing graft injury.
The role of the isoagglutinin (IA) titer in liver transplantation (LT) is still not well defined, but the general belief is that a higher titer may result in a higher risk of rejection [1-3]. To reduce the IA titer, plasmapheresis is usually performed 1-2 weeks before ABOi LDLT. However, due to the lack of sufficient evidence for the role of plasmapheresis, it is unclear whether plasmapheresis is necessary for all patients undergoing ABOi LDLT. Several studies have re- 


\section{HIGHLIGHTS}

- Plasmapheresis is usually performed before transplantation.

- Patients showed the high isoagglutinin titers even after plasmapheresis.

- The splenectomy and intravenous immunoglobulin were applied.

ported that sufficient DSZ for ABOi LDLT can be achieved using RTX alone [4-6]. In our center, if the baseline IA titer $(\leq 1: 32)$ is low, ABOi LDLT is carried out using conventional immunosuppression and RTX alone [4]. Otherwise, plasmapheresis combined with RTX is used as protocol to reduce the IA titer by 1:16 or lower before transplantation (Fig. 1). Our patients undergo conventional plasmapheresis (total plasma exchange), using freshly frozen blood type $A B$ plasma or albumin. One session of plasmapheresis is carried out 1-1.5 times at the predicted plasma volume every other day.

However, there is no established protocol for patients who have failed plasmapheresis before reaching target IA titers. The purpose of this case series is to show successful ABOi LDLT in patients who show high baseline IA titers and have failed plasmapheresis. The protocol of this retrospective cohort study was approved by the Institutional Review Board of Seoul National University Bundang Hospital, Seongnam, Korea, an academic hospital affiliated with Seoul National University, College of Medicine (IRB No. B-2003-603-702). Informed consent was waived because of the retrospective nature of the study and the analysis used anonymous clinical data.

\section{CASE REPORT}

\section{Case 1}

Non-response to plasmapheresis

The patient was a 47-year-old male $(\mathrm{Rh}+, 0)$ with hepatitis $B$ induced liver cirrhosis (LC) and hepatocellular carcinoma (HCC). Due to HCC recurrent, he was referred to our department for LT (Child-Pugh Score and model for end-stage liver disease [MELD] score was B7 and 9). His 52-year-old brother $(\mathrm{Rh}+\mathrm{A})$ was determined to be a ABOi living donor. Preoperative donor specific antibody (DSA) and cross-matching were negative. The patient received RTX $\left(300 \mathrm{mg} / \mathrm{m}^{2}\right) 3$ weeks before transplantation (percentage of cluster of differentiation 19 [CD19] decreased from $18 \%$ to $<1 \%$ ). The initial titers of immunoglobulin $G$ (IgG) and immunoglobulin M (IgM) were 1:256 and 1:64. The patient underwent conventional plasmapheresis using $5 \%$ albumin for whole replacement volume. After three plasmapheresis procedures, IA titers were not effectively reduced (IgG 1:256 and IgM 1:4). We decided to proceed with additional plasmapheresis procedures through multidisciplinary discussions. However, despite two additional cycles of plasmapheresis, the IA titer did not decrease to the target value ( $\operatorname{IgG} 1: 256$ and $\operatorname{IgM} 1: 8$ ). Splenectomy combined with intravenous immunoglobulin (IVIg; $0.8 \mathrm{~g} /$ $\mathrm{kg}$, from anhepatic phase to 2 days after transplantation) was done to reduce the possibility of rejection (Fig. 2). LDLT was completed without complications. The protocol biopsy on postoperative day (POD) 7 showed no histologic evidence of acute rejection. The titers of IgG vs. IgM were $1: 16$ vs. $1: 2$ and $1: 16$ vs $1: 1$ at POD 7 and 30 , respectively.

Case 2

Allergic reaction associated with plasmapheresis
Desensitization protoco

IA titer $(>1: 32) \rightarrow$ PP until $<1: 16$ IA titer $(\leq 1: 32) \rightarrow$ PP skip

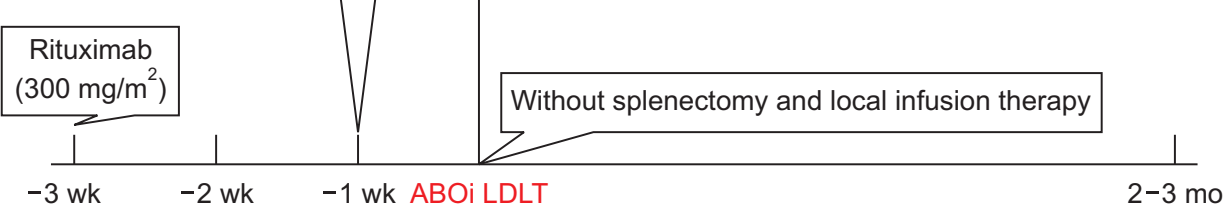

Fig. 1. Desensitization protocol for $A B O$ incompatible $(\mathrm{ABOi})$ living donor liver transplantation. LDLT, living donor liver transplantation; MMF, mycophenolate mofetil; IA isoagglutinin; PP, plasmapheresis. 


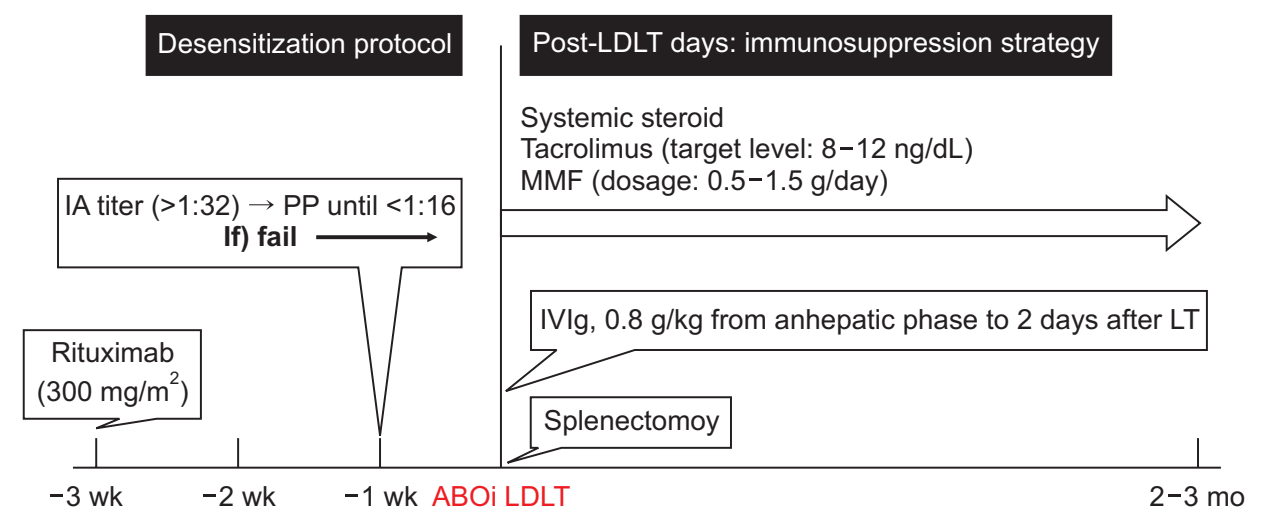

Fig. 2. Desensitization protocol for patients who have failed plasmapheresis. LDLT, living donor liver transplantation; MMF, mycophenolate mofetil; IA, isoagglutinin; PP, plasmapheresis; IVIg, intravenous immunoglobulin; LT, liver transplantation; ABOi, ABO incompatible.
The patient was a 58-year-old male $(\mathrm{Rh}+, 0)$ with hepatitis B induced LC and HCC. We decided to perform LT due to recurrent HCC (Child-Pugh Score and MELD score was B7 and 9). A possible living donor was his 54-yearold brother $(\mathrm{Rh}+, \mathrm{B})$. Preoperative DSA and cross-matching were negative. The percentage of $C D 19$ decreased from $37 \%$ to $1 \%$ after receiving RTX $\left(300 \mathrm{mg} / \mathrm{m}^{2}\right) 3$ weeks before transplantation. The initial titers of IgG and IgM were 1:512 and 1:64. An allergic reaction, such as itching sensation and hypotension, occurred during the first plasmapheresis, and so the procedure was interrupted. The cause was presumed to be the albumin used in the replacement fluid. To prevent the allergic reaction, the patient was administered with $100 \mathrm{mg}$ of hydrocortisone and $4 \mathrm{mg}$ of chlorpheniramine intravenously before starting the 2nd plasmapheresis. Fresh frozen plasma (FFP) was used as a replacement fluid. Despite the changes, it was discontinued due to an allergic reaction. Therefore, we decided to stop additional plasmapheresis. The final IgG and IgM titers before transplantation were 1:64 and 1:8. Splenectomy combined with IVIg was done. LDLT was completed without complications. The protocol biopsy on POD 7 were consistent with acute cellular rejection (ACR) of a mild degree (rejection activity index [RAI] score, 3). Even if there was an ACR finding with a RAI score of 3 , there was no sign of graft failure at the time, and rejection therapy was not performed. The titers of IgG vs. IgM were $1: 4$ vs. $1: 1$ and $1: 2$ vs. negative at POD 7 and 30 , respectively.

\section{Case 3}

Anaphylactic reaction to platelet transfusion

The patient was a 64-year-old male $(\mathrm{Rh}+, 0)$ with hepatitis B induced LC and HCC who had a history of platelet transfusion-related anaphylaxis. He was readmitted for
LT due to HCC recurrence and deteriorating liver function (Child-Pugh Score and MELD score was B7 and 16). His 19-year-old son $(\mathrm{Rh}+\mathrm{A})$ was determined to be a living donor. Preoperative DSA and cross-matching were negative. The patient received RTX $\left(300 \mathrm{mg} / \mathrm{m}^{2}\right) 3$ weeks before transplantation (percentage of $\mathrm{CD} 19$ decreased from $16 \%$ to $<1 \%$ ). The initial titers of IgG and IgM were $1: 128$ and 1:64. After the first cycle of plasmapheresis, IA titers were IgG 1:64 and $\operatorname{Ig} M$ 1:32. Platelet count decreased from $37 \times 10^{3} / \mu \mathrm{L}$ to $23 \times 10^{3} / \mu \mathrm{L}$. Because of previous allergic reactions, washed apheresis platelets were transfused. Fever, shivering and hypotension developed 1 hour after finishing the transfusion. The patient was managed with hydration and antipyretics. Even though the IgG titer did not reach the target value of 1:16, we decided not to perform any further plasmapheresis due to the burden of pancytopenia and coagulopathy that occurred after plasmapheresis. Splenectomy and IVIg administration were performed. The patient was transfused with 23 units of red blood cells, 27 units of FFP, and three units of apheresis platelet during surgery and no transfusion-related allergic reactions occurred. LDLT was completed without complications. The protocol biopsy on POD 7 showed no evidence of acute rejection. The titers of IgG vs. IgM were $1: 4$ vs. $1: 1$ and 1:2 vs. negative at POD 7 and 30 , respectively.

\section{DISCUSSION}

As reflected in the remarkable improvement in survival outcomes, ABOi LDLT has become an effective treatment option for end-stage liver disease. However, an effective DSZ protocol is the Achilles' heel of ABOi LDLT [7]. The 
adequate reduction of $B$ cells and the elimination of IA titers are important steps concerning the prevention of rejection [8]. However, DSZ protocols differ at each center, and the necessity of local infusion, splenectomy, IVIg, and plasmapheresis is controversial [9].

Although, the evidence for RTX monotherapy is still debated, our institution is selectively implementing RTX monotherapy when the baseline initial IA tier is low (<1:32) [4]. Otherwise, plasmapheresis combined with RTX is used as protocol to reduce the IA titer by 1:16 or lower. However, we identified patients who did not response to plasmapheresis or were unable to undergo plasmapheresis. There is no established protocol for patients who have failed plasmapheresis before reaching the target IA titers. Besides, Rummler et al. [10] reported that patients with HCC who had a high titer were at high risk for arterial thrombosis and graft loss. Therefore, effective immunosuppressive strategies were required to prevent acute rejection after transplantation.

Splenectomy in ABOi LDLT is often criticized for overwhelming postsplenectomy infections compounded by the aggressive immunosuppression used for ABOi LT $[11,12]$. In addition, several studies have reported that splenectomy had not decreased the incidence of antibody-mediated rejection [3]. Therefore, the prophylactic use of RTX without splenectomy in ABOi LDLT has emerged. However, for these patients, a strategy was needed to lower the IA titer before surgery. Here, we decided to perform a splenectomy because it is expected to eradicate remnant antibody-producing plasma cells that cannot be eliminated by plasmapheresis [13].

The mechanism of IVIg has been proposed to include the blocking of Fc receptors to non-nuclear cells, direct antibody neutralization, suppression of CD19 presentation to activated B cells, suppression of complements, and suppression of all T cells [14]. However, the optimal dose, timing, and frequency has not been established. In our center, IVIg was administered from anhepatic phase to 2 days after transplantation to prevent rebound increase.

Here, we have focused on successful ABOi LDLT cases using splenectomy and IVIg administration in patients for whom plasmapheresis failed. Patients had low IA titers after transplantation and showed no rebound rise. An important objective of this work is to demonstrate that we can apply the protocol mentioned above in patients who show high baseline IA titers and in whom plasmapheresis has failed. Furthermore, this report aims to promote this approach among the transplant community for the type of recipients described above.

\section{ACKNOWLEDGMENTS}

\section{Conflict of Interest}

No potential conflict of interest relevant to this article was reported.

\section{Funding/Support}

This study was supported by research grant from the Korean Society for Transplantation (2020-02-02001-006).

\section{ORCID}

Boram Lee https://orcid.org/0000-0003-1567-1774 Jai Young Cho https://orcid.org/0000-0002-1376-956X Hae Won Lee https://orcid.org/0000-0002-3312-9295 YoungRok Choi https://orcid.org/0000-0003-2408-7086 Yoo-Seok Yoon https://orcid.org/0000-0001-7621-8557 Ho-Seong Han https://orcid.org/0000-0001-9659-1260

\section{Author Contributions}

Conceptualization: BL, JYC. Data curation: BL, JYC, YRC, YSY, HSH. Formal analysis: all authors. Methodology: BL, JYC. Writing-Original draft: BL, JYC. Writing-review \& editing: $B L, J Y C, H W L$.

\section{REFERENCES}

1. Egawa $\mathrm{H}$, Teramukai $\mathrm{S}$, Haga $\mathrm{H}$, Tanabe $\mathrm{M}$, Mori $\mathrm{A}$, Ikegami $T$, et al. Impact of rituximab desensitization on blood-type-incompatible adult living donor liver transplantation: a Japanese multicenter study. Am J Transplant 2014;14:102-14.

2. Egawa $\mathrm{H}$, Teramukai $\mathrm{S}$, Haga $\mathrm{H}$, Tanabe $M$, Fukushima $M$, Shimazu M. Present status of ABO-incompatible living donor liver transplantation in Japan. Hepatology 2008;47:143-52.

3. Song GW, Lee SG, Hwang S, Kim KH, Ahn CS, Moon $\mathrm{DB}$, et al. ABO-incompatible adult living donor liver transplantation under the desensitization protocol with rituximab. Am J Transplant 2016;16:157-70.

4. Lee B, Choi Y, Han HS, Yoon YS, Cho JY, Jeong SH, et al. ABO-incompatible liver transplantation using only rituximab for patients with low anti-ABO antibody ti- 
ter. Ann Hepatobiliary Pancreat Surg 2019;23:211-8.

5. Lee EC, Kim SH, Shim JR, Park SJ. A comparison of desensitization methods: rituximab with/without plasmapheresis in ABO-incompatible living donor liver transplantation. Hepatobiliary Pancreat Dis Int 2018;17:119-25.

6. Yamamoto H, Uchida K, Kawabata S, Isono K, Miura K, Hayashida S, et al. Feasibility of monotherapy by rituximab without additional desensitization in ABO-incompatible living-donor liver transplantation. Transplantation 2018;102:97-104.

7. Yadav DK, Hua YF, Bai X, Lou J, Que R, Gao S, et al. $A B O-$ incompatible adult living donor liver transplantation in the era of rituximab: a systematic review and meta-analysis. Gastroenterol Res Pract 2019;2019:8589402.

8. Lee CF, Cheng $\mathrm{CH}$, Wang YC, Soong RS, Wu TH, Chou $\mathrm{HS}$, et al. Adult living donor liver transplantation across ABO-incompatibility. Medicine (Baltimore) 2015;94:e1796.

9. Shen T, Lin BY, Jia JJ, Wang ZY, Wang L, Ling Q, et al. A modified protocol with rituximab and intravenous immunoglobulin in emergent $A B O$-incompatible liver transplantation for acute liver failure. Hepatobiliary Pancreat Dis Int 2014;13:395-401.

10. Rummler S, Bauschke A, Baerthel E, Juette H, Maier K, Malessa $C$, et al. ABO-incompatible living donor liver transplantation in focus of antibody rebound. Transfus Med Hemother 2017;44:46-51.

11. Troisi R, Hesse UJ, Decruyenaere J, Morelli MC, Palazzo U, Pattyn P, et al. Functional, life-threatening disorders and splenectomy following liver transplantation. Clin Transplant 1999;13:380-8.

12. Usui M, Isaji $S$, Mizuno $S$, Sakurai $H$, Uemoto $S$. Experiences and problems pre-operative anti-CD20 monoclonal antibody infusion therapy with splenectomy and plasma exchange for $\mathrm{ABO}$-incompatible living-donor liver transplantation. Clin Transplant 2007;21:24-31.

13. Kawagishi N, Satomi S. Current aspects of ABO-incompatible liver transplantation. Clin Case Rep Rev 2016;2:375-9.

14. Kim JD, Choi DL, Kim SG, Lee AJ. Single-center experience of $\mathrm{ABO}$-incompatible living-donor liver transplantation with a new simplified intravenous immunoglobulin protocol: a propensity score-matching analysis. Transplant Proc 2016;48:1134-8. 